\title{
LE FEU ET SES USAGES MILITAIRES
}

PAR

J.F. FINO

LE feu a toujours été une arme puissante. L'homme préhistorique, les Assyriens, les Grecs, les Byzantins, les Arabes, les bombes incendiaires de notre aviation, autant d'exemples de son usage militaire dans des pays et des temps fort divers. De plus, une de ses variantes, le feu grégeois pulvérulent, a été à l'origine de la poudre à canon. Il a donc paru intéressant de rassembler quelques données à ce sujet et de rappeler briévement la production des feux de guerre, leur lancement et la défense contre ses effets. Accessoirement, de dire quelques mots sur une autre branche de la chimie de guerre: les gaz asphyxiants ${ }^{1}$.

\section{LA PRODUCTION DU FEU}

Le feu peut être transmis par contact, au moyen de projectiles de pierre, d'argile, etc., ou même par du sable, suffissaments chauffés. Lors du siège de Marseille para les troupes de César les défenseurs auraient ainsi lancés avec les balistes des barres de métal rougies au feu qui enflammaient le bois des ouvrages ennemis ${ }^{2}$. Aux siècles passés, un procédé analogue a été employé sous le nom de «boulets rouges» et dans plusieurs musées d'Europe il est donné de voir des appareils servant à cet usage: sorte de rigole de fer, enroulée en spirale, sur laquelle glissent les boulets qu'un braséro central permet de chauffer convenablement.

Une technique plus directe consiste à mettre la flamme en contact avec l'objet à incendier. Lors des fouilles du palais assyrien de Khorsabad (VIII ${ }^{\circ}$ siècle avant J. C.) on a trouvé des bas-reliefs montrants que les assiégéants ainsi que les assiégés se servaient de projectiles incendiaires.

${ }^{1}$ Parmis les nombreux travaux relatifs au feux de guerre, nous indiquerons seulement: M. Mercter, Le feu grégeois, les feux de guerre depuis l'Antiquité, la poudre à canon, Paris, Geuthner, 1952, VI, 164 p. et XV pl.; J. R. Partington, A bistory of Greek Fire and gun powder, Cambridge, W. Heffner, 1960, 381 p. Deux contributions, plus anciennes, ne doivent pas être négligées: M. BERTHELOT, Les compositions incendiaires dans l'Antiquité et au Moyen Âge, le feu grégeois et les origines de la poudre à canon, in: «Revue des Deux Mondes», vol. 106 (1891), pp. 786-822; J. T. Reinaud et I. Favé, Du feu grégeois, des feux de guerre et des origines de la poudre à canon, Paris, J. Dumaine, 1845,285 p. et 1 album de XVII pl.

${ }^{2}$ Vitruve, De l'architecture, liv. X, chap. 16, éd. Nisard, Paris, Didot, 1852, p. 178. 
Sur la colonne Antonine ou Aurélienne (fin du $\mathrm{II}^{\mathrm{c}}$ siècle de notre Ėre) on voit l'attaque d'une redoute par les troupes romaines et un soldat qui, torche en main, se tient prêt à mettre le feu à l'ouvrage. La broderie de Bayeux (extrème fin du XI ${ }^{c}$ siècle, début du $\mathrm{XII}^{\mathrm{c}}$ ), en figurant la prise du château de Dinan, montre deux guerriers armés de torches qui s'efforcent de brûler la palissade formant la première enceinte de la forteresse.

Ces torches devaient consister, probablement, en des morceaux de bois imbibés de résine, de poix, de suif, etc. Mais ces produits peuvent être contenus aussi dans des vases de verre ou de terre-cuite qui, en se briasant contre l'obstacle, répandent l'incendie et les auteurs grecs ou latins font, assez souvent, mention de cette technique. Pour être vraiment efficace, le mélange doit réunir certaines conditions: s'enflammer à basse température, dégager beaucoup de chaleur pendant sa combustion, être gluant, visqueux, à fin de coller contre l'objet à incendier. A la poix, au soufre, au suif, on ajoute du naphte ou pétrole brut, fournit par les gissements d'Asie Mineure. Les peuples d'Orient, en particulier les Mèdes, semblent avoir utilisé ce produit depuis des temps reculés et le nom d'huile médique restera longtemps attaché au naphte. Au IV siècle de notre Ėre, Ammien Marcellin écrit: «C'est chez ce peuple [les Mèdes] que se confectionne l'huile médique. La flèche qu'on en imprège brûle tout objet auquel elle s'attache, pourvu qu'elle soit décochée mollement d'un arc à la corde lâche; car un jet trop rapide annule toute la vertu de la composition. L'eau qu'on emploirait pour étteindre ce feu ne ferait que le rendre plus intense; on n'en triomphe qu'en l'étouffant sous le sable. Voici la recette de cette huile: on prend des feuilles d'une certaine herbe que l'on laisse macérer dans de l'huile commune; et quand la solution est opérée, on épaissit le résidu avec une substance qui ressemble à de l'huile densé production naturelle du sol, avons nous dit, et qu'on appelle naphte en langue du pays» ${ }^{3}$.

L'addition d'huile de térébenthine puis d'huile de genièvre - préparées au moyen d'alambics, appareils déjà connus au $\mathrm{III}^{\mathrm{e}}$ siècle ${ }^{4}$ renforce l'efficacité du mélange, ces huiles étant éminemment inflammables et le feu qu'elle provoquent difficile à maitriser.

Toutefois ces préparations ne peuvent être lancées trop vivement, sous peine de d'éteindre. Ammien Marcellin le note spécialement dans le morceau cité antérieurement ainsi que dans un autre endroit de son oeuvre transcript plus loin. Ce «lancer mou» réduit la portée du projec-

'Ammien Marcellin, liv. XXIII, chap. 6, éd. Nisard, Paris, Didot, 1869, p. 204.

+ Des alambics primitifs sont reproduits dans le vol. I de M. Berthelot, Collection des anciens alchimistes grecs, 3 vols., Paris, G. Steinheill, 1887-1888. 
tile. L'addition de salpêtre et de chaux vive constitue le véritable progrès. Sans chercher à établir ici quels furent les auteurs probables de l'innovation et tout en signalant que l'usage du salpêtre à des fins militaires semble avoir été plutôt tardif chez les Arabes ${ }^{5}$, nous nous bornerons à constater qu'il constitue un ingrédient précieux. En effet, le salpêtre ou azotate de potassium, en se décomposant sous l'action de la chaleur, dégage une grande quantité d'oxygène, ce qui rend la combustion indépendante de l'apport de l'oxygène atmosphérique et il n'est point facile d'étouffer un feu ainsi alimenté. De plus, le projectile pouvant être lancé avec une forte vitesse initiale, sa portée s'en trouve singulièrement accrue. Quant à l'adjonction de chaux vive, soit seule soit mélée à divers produits formant pyrophore, elle permet d'obtenir un feu s'allumant en présence de l'eau ce qui outre des avantages spécifiques, cause un effet psychologique intense.

Les Byzantins se servent courament des feux de guerre qui, selon la tradition, auraient été introduits chez eux par «l'architecte» Callinique, au VII ${ }^{\mathrm{c}}$ siècle 6 . La possesion du «feu grégeois» (nom appliqué aux divers produits sortis des laboratoires de Byzance) leur assure une nette supériorité militaire sur leurs voisins et ils peuvent résister ainsi aux attaques des Russes, des Arabes et des autres peuples de l'Orient. Ces mélanges complexes, dont la composition a du changer au cours des siècles et selon les opérateurs, sont formés des produits habituels - résine, graisses, huiles, etc. - et les Byzantins n'en font point mystère. Par contre, tout ce qui a trait à l'addition de la chaux et du salpêtre, est considéré comme un secret d'État, dont la connaissance est réservée à quelques initiés. Au $\mathrm{X}^{\mathrm{c}}$ siècle, l'empereur Constantin Porphyrogénète rappele dans son traité De l'administration de l'empire: «Tu dois pardessus toutes choses porter tes soins et ton attention sur le feu liquide

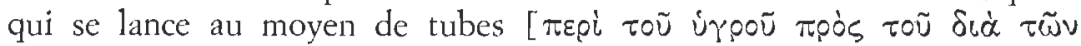

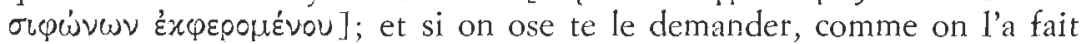
souvent à nous-même, tu dois repousser et rejeter cette prière en répondant que ce feu a été montré et révélé par un ange au grand et saint premier empereur chrétien Constantin [dans le chap. XLVIII du même traité, l'auteur se démentant lui-même, attribue l'invention du feu liquide à Callinique]. Par ce message et par l'ange lui-même, il lui fut enjoint, selon le témoignage authentique de nos pères et de nos ancêtres, de ne préparer ce feu que pour les seuls chrétiens, dans la seule ville impériale, et jamais ailleurs; de ne le transmettre et de ne l'enseigner

${ }^{5}$ Quelques mots à ce sujet sont dit in J.F. Finó, Forteresses de la France médiévale; construction, attaque, défense, $2^{\mathrm{c}}$ éd., Paris, Picard, 1970, pp. 209-210.

"A. BruHN HofrMeYer, Military equipment in the byzantine manuscript of Scylitzes in Biblioteca Nacional in Madrid, in: Glablus, vol. V (1966), pp. 143-145. 
jamais à aucune autre nation, quelle qu'elle fût. Le grand empereur alors, pour se précautionner contre ses successeurs, fit graver sur la sainte table de l'église de Dieu des imprécations contre celui qui oserait le communiquer à l'étranger. Il prescrivit que le traître fût regardé comme indigne du nom de chrétien, de toute charge et de tout honneur; que s'il avait quelque dignité il en fût dépouillé. Il déclara anathème dans les siècles des siècles, il déclara infâme quiconque, empereur, patriarche, prince ou sujet, aurait essayé de violer cette loi. Il ordonna en outre à tous les hommes ayant la crainte et l'amour de Dieu de traiter le prévaricateur comme un ennemi public, de le condamner et de le livrer au plus affreux supplice. Pourtant une fois il arriva [le crime se glissant toujours partout], que l'un des grands de l'Empire, gagné par d'immenses présents, communiqua ce feu à un étranger; mais Dieu ne put voir un pareil forfait impuni, et un jour que le coupable était près d'entrer dans la sainte Église du Seigneur, une flamme descendue du ciel l'enveloppa et le dévora. Tous les esprits furent saisis de terreur, et nul n'osa, désormais, quel que fût son rang, projeter et encore moins exécuter un crime aussi grand» ${ }^{7}$.

En 1204, lors de la IV ${ }^{\mathrm{e}}$ Croisade, Constantinople est prise par les armées franco-vénitiennes. La connaissance de ces formules se répand alors dans le monde latin. Le Liber ignium ad comburendos bostes de Marchus Graechus en est un exemple. C'est un manuel de praticien dont on ne connait que la version latine mais dont la rédaction, pleine de grécicismes, semble attester une origine byzantine ${ }^{8}$. Notons en passant que le Liber ignium prouve que la prétendue perte du secret du «feu grégeois» n'est qu'une légende, trop souvent répétée. Marchus Graechus indique des procédés pour raffiner le salpêtre, pour préparer divers feux grégeois et même pour fabriquer une véritable poudre noire servant à charger des pétards et des fusées.

«12. Une partie de colophane, autant de soufre vif, six (?) parties de salpêtre. Après avoir bien pulvérisé et imbibé d'huile, délayez dans l'huile de lin, ou plutôt dans l'huile de laurier. Ensuite, introduisez

${ }^{7}$ Constantin Porphyrogénète: De administrando imperio, in: A. Banduri, Imperium Orientale, Paris, 1711, vol. I, p. 64. Nous suivons la traduction donnée in M. Mercier, Le leu grégeois, p. 39.

${ }^{8}$ Marchus Graechus, Liber ignium ad comburendos bostes. Texte transmis par divers manuscrits: Biblioth. Nat. Paris, ms. 7156 (fin du XIII' siècle, début du $\mathrm{XIV}^{\mathrm{c}}$ ) et ms. 7158 (du XVc, copie du précédent?); Bayerische Staatsbl. Munich, ms. 267 (similaire au 7156) et ms. 197 (écrit vers 1438, rédaction très différente). Publié par F. J. G. LA Porte du Theil, Paris, Delance et Lesueur, 1804, VI, 18 p., puis par M. Berthelot, in: Histoire des sciences, la chimie au Moyen Áge, Paris, Imp. Nat., 1893, vol. I, pp. 89-135, avec les variantes des manuscrits de Paris et de Munich. 
dans un jonc ou dans un bâton creux et allumez. Il s'envole soudain vers l'endroit que vous voulez et incendie tout.

$\gg 13$. La seconde espèce de fusée se fabrique ainsi: un livre de soufre vif, deux livres de charbon de tilleul ou de saule, six livres de salpêtre. Ces trois choses seront pulvérisées très finement sur un marbre; puis vous mettrez la poudre à volonté dans une enveloppe de fusée ou de pétard. Notez. L'enveloppe de fusée doit être mince et longue et remplie avec de la poudre bien tassée. L'enveloppe de pétard doit être courte et épaisse, remplie à moitié de poudre et fortement liée par un fil de fer aux deux extrémités. Notez. Dans l'une et l'autre enveloppe, on doit pratiquer une petite ouverture où l'on place une mèche pour mettre le feu. Cette mèche sera mince aux extrémités, au milieu large et remplie de la poudre ci-dessus. Notez. L'enveloppe de la fusée peut avoir plusieurs tours, celle du pétard le plus possible.»

On peut ranger les feux grégeois en trois groupes principaux selon qu'il consistent dans des mélanges liquides, pâteux ou solides. Dans les deux premiers cas, le carbone nécéssaire à la combustion est fourni par le naphte, les graisses, etc.; dans le troisième par le charbon de bois tendre. Le résultat est comparable, mais les mélanges solides de soufrc, de salpêtre et de charbon de bois - le mercure, la limaille de fer, l'arsénic sulfuré, etc., pouvant lui être additionné - déplaceront les deux autres lorsqu'on aura l'idée d'en utiliser la force «projective» pour le lancement des projectiles. Ils s'imposeront universellement et avec eux l'indispensable charbon de bois. Ce n'est qu'au début du $\mathrm{XX}^{\mathrm{c}}$ siècle que, par une sorte de retour, les benzols - liquides dérivés du pétrole prènent la place du charbon de bois et fournissent les explosifs modernes: mélinite, tolite, etc.

Mais, et ceci est important, les divers moyens de produire les feux coéxisteront longtemps entre-eux, au hasard des régions ou de la science des «engineors». En effet, la formation de ceux-ci est essentiellement pratique acquise sur le chantier, formée de tours de main transmis de maître à élève. Quant aux «réceptaires», qui s'échelonnent tout le long du Moyen Âge, leur interêt est indéniable mais il s'agit de compilation de formules, parfois extrêmement anciennes, recopiées de siècle en siècle et auxquelles le posseseur occasionnel se contente parfois d'ajouter les recettes qu'il appliqué lui-même, sans qu'il soit procédé à une refonte générale?. Il n'y a donc point de progrès linéaire, universel, et un seul exemple suffit à le prouver. En 1216, en plein XIII" siècle, lors du siège du château de Beaucaire, les défenseurs font appel à un «engineor» qui, pour incendier une chatte des ennemis, prend un pot, le rempli

${ }^{9}$ J.F. Finó, Forteresses de la France médiévale, $2^{\mathrm{e}}$ éd., pp. 11-16. 
«d'alquitran» (poix ou goudron) et le lance enflammé contre l'engin ${ }^{10}$. Pour efficace qu'il fut, le projectile n'en ressortissait pas moins d'une technique toute primitive.

\section{LANCEMENT DES FEUX}

Le naphte et les matière grasses qui entrent dans la composition du feu grégeois font flotter celui-ci sur la surface de la mer, ce qui suffit

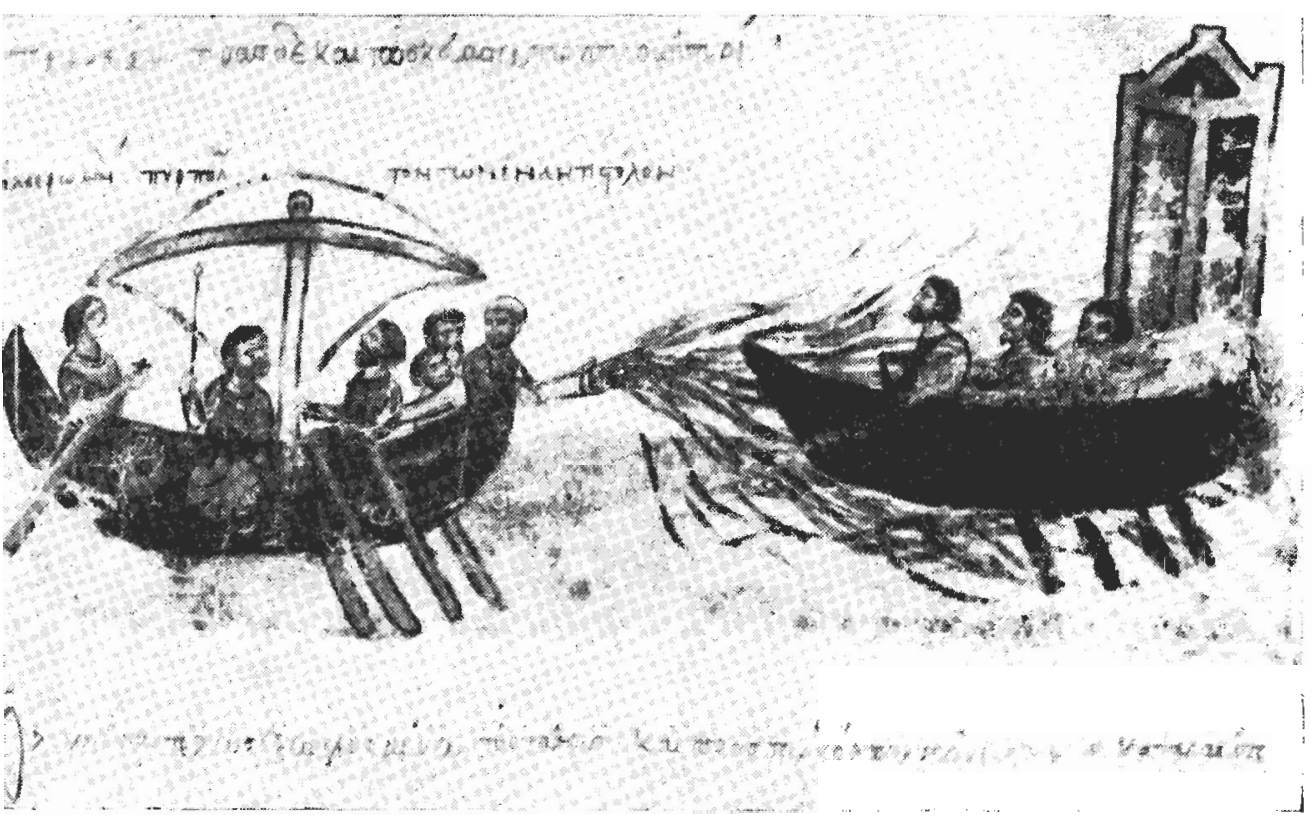

FIg. 1.-Lancement du feu grégeois au cours d'une halaille navale. Miniature byzantine du manuscrit du Scylitzes (Bibl. Nacional, Madrid, Cod. Matritensis, vit. 26-2. fol. $\left.34^{\circ}\right)$. (Cl. A. Bruhn de Hoffmeyer.)

à la défense des côtes et des ports. Récemment, Mr. Geoffroy Lloyd a révélé qu'en 1940, lorsque l'Angleterre était menacée d'invasion, de grands réservoirs pleins de pétrole avaient été placés près du rivage. Le

${ }^{10}$ La chanson de la croisade albigeoise, laisse 167, vers 13-17, éd. E. MartinChabot, Paris, Champion-Les Belles Lettres, 1931-1961, vol. II, pp. 172-173. 
cas échéanf, leurs contennu aurait été déversé et, enflammé par du phosphure de calcium, se serait répandu sur les lieux de débarquement ${ }^{11}$.

Sur terre on pouvait procéder d'une façon similaire. Une partie du champ de bataille étant imbibée de naphte, on y méttait le feu après y avoir attiré l'ennemi. Les textes font parfois mention d'un tel stratagème qui dut être employé assez fréquemment.

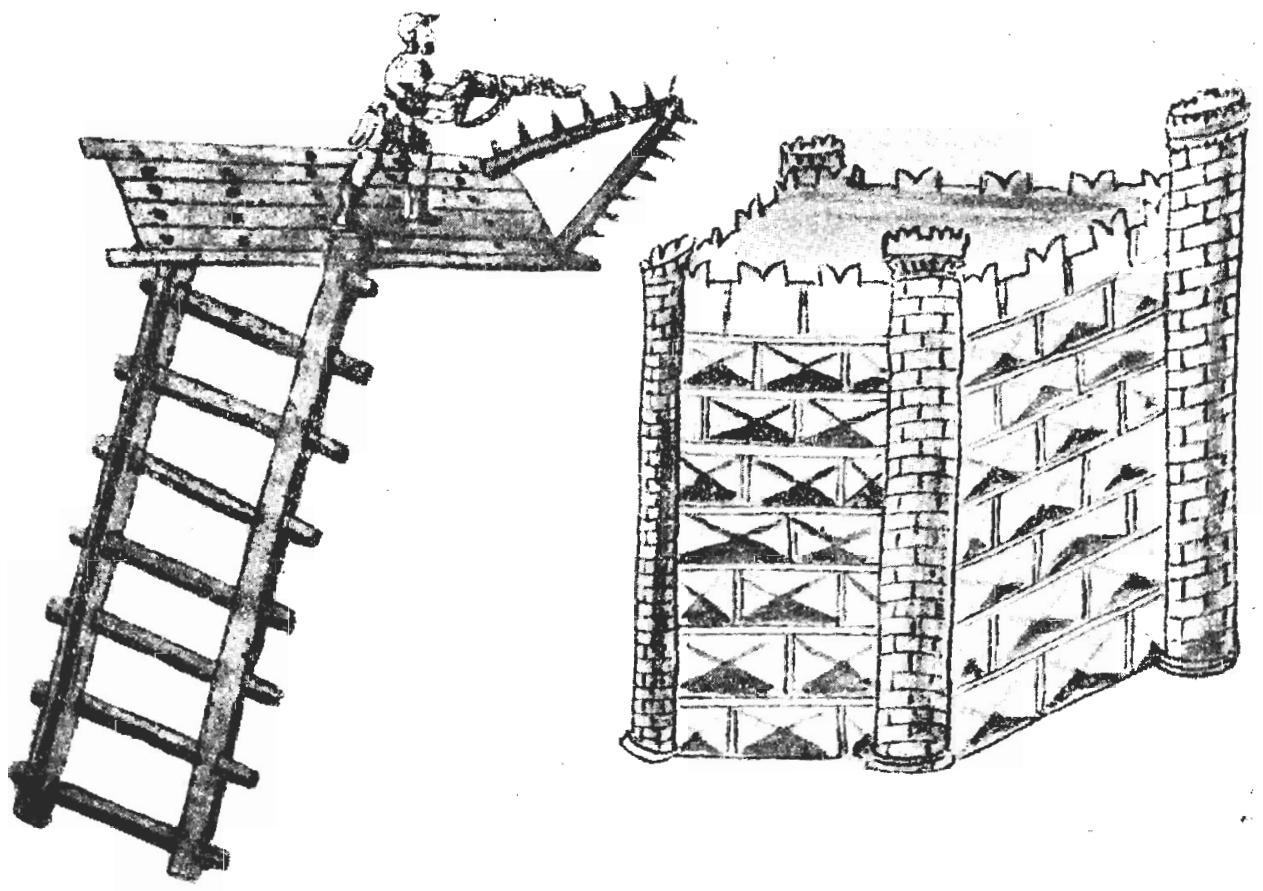

Fig. 2.-Lancement du feu grégeois lors de l'attaque d'une forteresse. Miniature d'un manuscrit byzantin de la Bibl. de Bologne. La même scène est reproduite dans un manuscrit de la Bibl. Vaticane (Cod. grec. 1605). (Cl. A. Bruhn de Hoffmeyer.)

Dans les rencontres navales, les Byzantins projettent le feu grégeois au moyen d'un tube de métal placé à l'avant des navires, le produit étant refoulé par une pompe. Des fragments de pompes métalliques

" La note de M. Lloyd se trouve reproduite in M. MERCIER, Le feu grégeois, pp. 128-129. 
trouvés au cours de fouilles, prouvent que celles-ci étaient déjà connues des Romains ${ }^{12}$ et une miniature byzantine montre la scène ${ }^{13}$. Pour les opérations terrestres, par example, l'attaque d'une forteresse, le feu peut être lancé par une sorte de seringue, tel qu'il apparait dans d'autres miniatures ${ }^{14}$.

Lorsque les mélanges inflammables sont placés à l'intérieur d'un manche creux, on a les «massues à asperger», les «bâtons à feu» et même de véritables lance-flammes. Ces armes, courament utilisés par les Byzantins et par les Arabes, sont redoutables et de nos jours elles l'ont bien prouvé sur les champs de bataille de l'Europe ou de l'Asie.

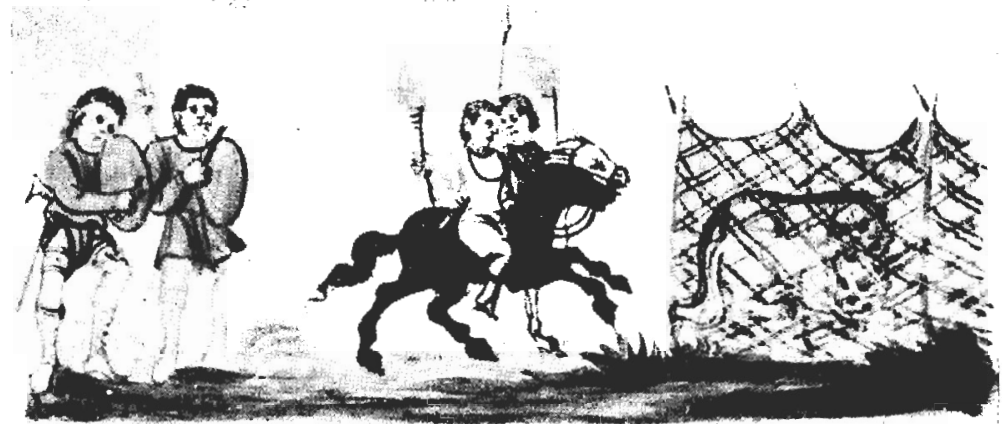

FIG. 3.-Bátons à feu utilisés par des cavaliers. Miniature byzantine de Ja Cynégétique d'Oppien (Bibl. Nationale, Paris, grec n." 2736, fol. 51).

(Cl. Biblioth. Nation.)

Si le produit est contenu dans un récipient porté par une hampe, on a une variante du procédé antérieur. Mais le récipient peut être lancé aux loin, soit à la main, soit à l'aide d'une fronde. Divers musées d'Europe et du Proche Orient conservent un certain nombre de petits vases, d'origine orientale, faits en terre-cuite et façonnés manuellement

12 Une reconstruction de la pompe romaine trouvée à Bolsena (Italie), faite d'après les fragments conservés au British Museum, a été reproduite par R. J. ForBES et H. S. Goodricir, in World Petroleum, mai 1939; par A. R. HALL, in A bistory of technology, 2éd., Oxford, Clarendon Press, 1957, vol. II, p. 376; par M. MerCIER, in Le feu grégeois, face p. 28; etc.

${ }_{13}$ A. Bruhn Hoffmeyer, Military equipment..., in: Gladius, vol. V (1966). fjg. 24, qui restitue l'appartenance de la miniature au manuscrit de Madrid.

14 Ibid., fig. 21. 
ou au tour, d'après un modèle plus ou moins standardisé. Ils sont à rapprocher de ceux qui aparaissent figurés dans les miniatures de l'artificier arabe al-Hasan al-Rammah Nağim al-din al-Ahdab et nul doute qu'ils n'aient été destinés au lancement de mélanges inflammables ${ }^{15}$.

Fig. 4.- Pot à napbie arabe. Trouvé à Fostat (Egypte) il fait partie de la collection Mercier. En terre cuite, son poids est de 460 gr., sa contenance de 345 gr. d'eau et ses dimensions 115 millimètres de hauteur par 105 de dia. mètre. Probablement lancé à la main. (Collection Mercier.)

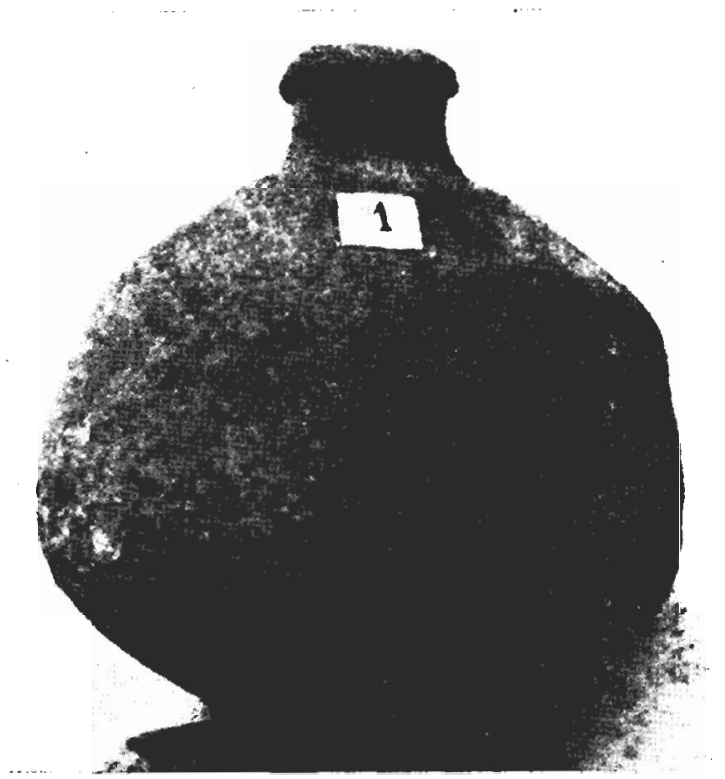

Les pots de dimensions plus grandes sont lancés au moyen de machines de jet. Joinville, bon observateur dans sa description de la VII Croisade (1250) à laquelle il participa, écrit: «Un soir... il advint qu'ils [les Sarrazins] nous amenèrent un engin que l'on appelle pierrière, ce qu'ils n'avaient pas encore fait, et ils mirent le feu grégeois dans la

${ }^{15}$ Nedjm Eddin Hassan Alramimah (ou El Rammah), Traité de combattre à cheval et des machines de guerre. Texte transmis par divers manuscrits de la Bibliot. Nat. de Paris: fond oriental, n. 2825 (ancien, n. ${ }^{\circ} 1127$ ); avec peintures, n." 2827 (ancien fond Asselin, n. ${ }^{\circ}$ 643); sans peintures, n. 2829 (ancien supplément des ms. arabes, n. ${ }^{\circ} 733$ ), contient le texte de Hassan mais attribué à un autre auteur. Echantillonnage de recettes et de figures souvent reproduits, p. e., in: J. T. Reinaud et I. Favé, Du feu grégeois, pp. 20-51, et Atlas, pl. I-III; L. N. Bonaparte, Études sur le passé et l'avenir de l'artillerie, Paris, J. Dumaine, 1846-1871, vol. III, pp. 20-24 et pl. 1-2. 


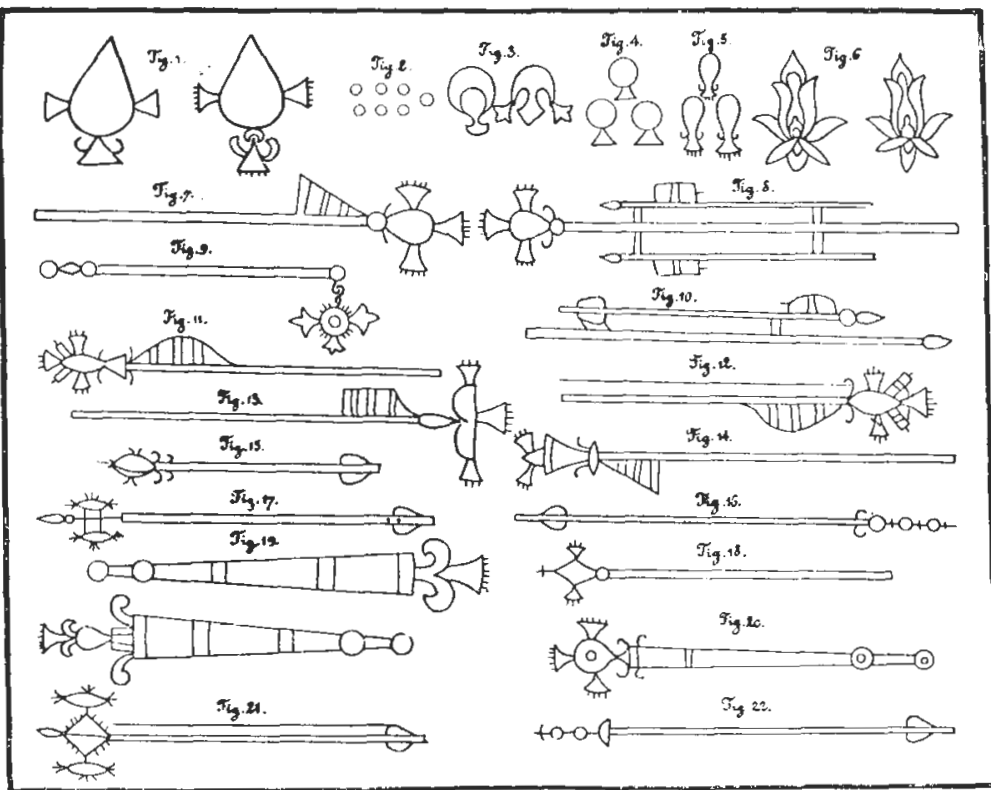

Fig. 5.--Récipients et projectiles arabes pour le lancement des feux de guerre. Dessins de J. T. Reinaud et I. Favé (Du feu grégeois, atlas, pl. III) d'après le Traité de Hassan Alrammah. Sur la première rangée horizontale sont figurés divers modèles de récipients qu'une erreur du dessinateur a placé la tête en bas. Puis des flèches avec une ou deux fusées porteuses latérales et munies ou non de vase pout le feu grégeois pâteux. Enfin, des lances à feu, des massue à asperger, etc.

fronde de l'engin» ${ }^{16}$. Les ingénieurs militaires du $X V^{\mathfrak{e}}$ siècle parlent souvent de trébuchets utilisés pour le lancement de barils remplis de produits incendiaires et même d'animaux ayant un récipient enflammé attaché à son dos, ce qui permet de répandre l'incendie ${ }^{17}$. Le manuscrit dit «de la guerre hussiste» ${ }^{18}$ contient, au fol. $28^{\mathrm{v}}$, une miniature repré-

${ }^{16}$ Jean DE JoInville, Histoire de Saint-Louis, chap. 43, $\$ 203$, éd. N. de Wailly, Paris, Didot, 1874, pp. 112-113.

${ }_{17}$ B. Gille, Les ingénicurs de la Renaissance, Paris, Hermann, 1964, 238 p., donne un catalogue de leurs manuscrits.

${ }_{18}$ Bayerische Staatsbibl. Munich, ms. latin 197. Les figures sont reproduites in M. Berthelot, Pour l'bisioire de l'artillerie el des arts mécaniques vers la fin du Moyen Âge, in: «Annales de chimie et de physique», t. 24 (1891), pp. 433-521. 
sentant un cheval porte-feu et il est difficile de ne pas évoquer alors le Samson biblique lançant contre les Phillistins trois cents renards portant, chacun, un flambeau attaché à la queue.

Joinville nous dit aussi: «Trois fois ils [les Sarrazins] nous jetèrent le feu grégeois ce soir-là, et ils nous le lancèrent quatre fois avec l'arbalète à tour» ${ }^{19}$. Il y a lieu de remarquer ici que les arbalètes à tour lançant des dards ou des javelots, ces projectiles pouvaient être remplacés par des tisons ou des flèches de bois enflammé. Mais lorsqu'on se sert de véritables produits incendiaires, ceux-ci doivent impregner une étoupe enroulée autour de la hampe. On a ainsi les malleoli et les phalariques que Végèce décrit à la fin du IV siècle: «On lance avec de grandes balistes des marteaux [malleoli] ou des phalariques qui percent les peaux et les couvertures et portent le feu dans le bois. Les marteaux sont des sortes de flèches ardentes qui mettent le feu partout où elles peuvent s'attacher. La phalarique est une espèce de lance armée d'un gros fer entre lequel et la hampe on entortille des étoupes pleines de soufre, de bitume, de résine et d'huile incendiaire» ${ }^{20}$. Pour sa part, Ammien Marcellin dit: «Le projectile appelé malleolus est une flèche de roseau garnie tout autour de bandes de fer qui se renflent par le milieu, en laissant ouverts leurs interstices, ce qui lui donne l'apparence extérieure d'un fuseau à filer. La concavité se remplit de matières inflammables aux-quelles on met le feu; et ce trait, lancé par un arc à la corde lâche (une vibration vigoureuse l'éteindrait) brûle avec opiniatreté tout corps auquel il s'attache. L'eau même ne fait qu'accroitre l'intensité de l'embrassement; et ce n'est qu'en jetant dessus de la poussière qu'on parvient à le maîtriset» ${ }^{21}$.

A en croire le chroniqueur Jean, moine de Marmoutier, la lecture de Végèce aurait inspiré à Geoffroy IV le Bel, dit Plantagenêt, la fabrication d'un projectile incendiaire lancé contre le château de MontreuilBelay qu'il assiège en $1151^{22}$. Le Musée de l'Armée de Paris, sous les cotes L-1 à L-3 présente deux arcs d'environ $2 \mathrm{~m}$., probablement destinés aux arbalètes à toux, et deux traits, longs de $1 \mathrm{~m}$., munis de rondelles et d'anneaux pour y fixer la filasse imprégnée de feu grégeois. Ces objets, qui datent du XIV eu du XV siècle, doivent être d'origine orien-

19 JeAn de Jornville, Histoire de Saint-Louis, chap. 43, \$206, éd. N. de Wailly, pp. 112-113.

20. VÉGĖCE, Des institutions militaires, liv. IV, chap. 18, éd. Nisard, Paris, Didot, 1869, p. 723.

21 Ammien Marceilin, liv. XXIII, chap. 4, éd. Nisard, Paris, Didot, 1869, p. 196.

${ }^{22}$ Chronique des comles d'Anjou et des seigneurs d'Ambroise, éd. L. Halphen et R. Poupardin, Paris, Picard, 1913, pp. 217-218. 
tale ${ }^{23}$. En plein XVII ${ }^{\mathrm{e}}$ siècle, l'espagnol Diego Ufano, dans son Tratado della [sic] artillería... platicado en las guerras de Flandres, décrit des flèches porteuses de mélanges incendiaires à base de salpêtre, de soufre, de résine, de poix, d'huiles, etc., lancées au moyen de puissantes arbalètes ${ }^{24}$.

Lorsque des mélanges solides, pulvérulents, sont placés dans une sorte de cartouche mise à l'extrémité d'une flèche, ils agissent à la façon d'une fusée et la force de «réaction» oblige à placer la cartouche de sorte que le jet de flamme soit dardé vers l'arrière: si le projectile se fiche

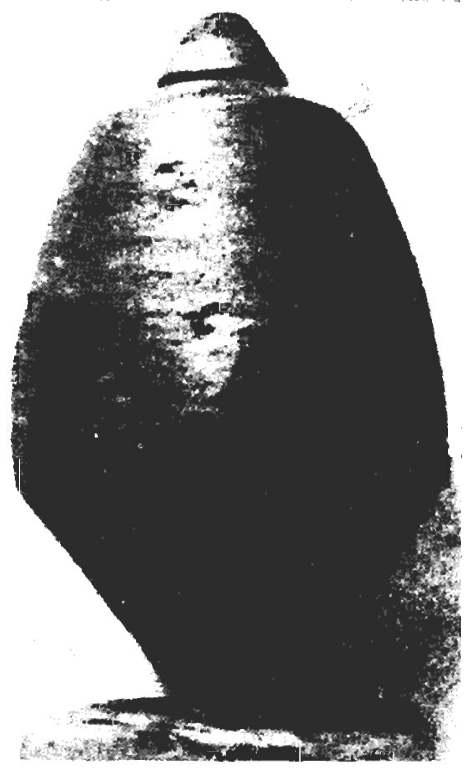

Fig. 6.-Grenade explosive arabe. Trouvée à Fostat, elle fait partie de la collection Mercier. En terre cuite, son poids est de 365 gr., sa contenance d'environ 175 à $180 \mathrm{gr}$. d'cau et ses dimensiones $131 \mathrm{~mm}$. de hauteur par $80 \mathrm{~mm}$. de diamètre. Le goulot, situé à l'extrémité supérieure du «chapeau», est fort étroit ( $2 \mathrm{~mm}$. environ). Lancement probable à la fronde ou moyennant une cordelette. (Cl. Mercier.)

contre un obstacle, le jet ne le touche pas. En outre, ces produits manquent de viscosité et ils n'adhèrent pas à l'objet à enflammer. On utilise donc conjointement les deux sortes de feux grégeois: le pulvérulent, allumé dès le départ, pour transmettre le feu et agir sur la flèche; le pâteux, enflammé seulement au bout de course, pour incendier l'obstacle.

${ }^{23}$ L. Robert, Catalogue des collections composant le Musée d'Artillerie en 1889, Paris, Imp. Nat., 1889-1893, vol. III, p. 377.

${ }^{24}$ L'ouvrage de Diego Ufano fut publié à Bruxelles en 1613. Version française due à Th. de Bry, sous le titre Vraye instruction de l'artillerye, Frankfurt am Main, 1615. 
Sous le nom de «flèche de Kathay», les Arabes utilisent ces sortes de misiles et les miniatures du manuscrit de Hassan Alrammah montrent les deux sortes de projectiles, porteurs ou non d'un récipient contenant le feu grégeois pâteux. Enfin, l'on pratique assez fréquemment le procédé consistant à lancer d'abord des produits combustibles qui imprègnent l'ouvrage à détruire, puis à enflammer le tout ensemble afin d'obtenir un incendie de vastes proportions.

Il se pourrait aussi que les Arabes aient connue la force «explosive» des mélanges solides. Hassan Alrammah n'en parle point expressement mais il indique les précautions à prendre contre les dangers d'une explosion intempestive lors de leur maniement. Au cours de ses recherches, Mercier a repéré un certain nombre de petits vases, faits au tour, en série, d'une contenance d'environ $200 \mathrm{~cm}^{3}$, dont le goulot, très étroit, devait rendre difficile le remplissage avec un produit visqueux. L'auteur pense que ces récipient devaient être lancés contre l'ennemi, mèche allumée, et que les éclats vulnérants produits par leur explosion en faisaient de véritables grenades, similaires à celles utilisées lors de la guerre de $1914^{25}$.

\section{DÉFENSE CONTRE LES FEUX}

Le feu est, nous le répétons, une arme puissante et comme dans l'Europe du Haut Moyen Âge la plupart des ouvrages de défense sont de bois, il est curieux que son usage ne se soit pas répandu davantage en Occident tout comme chez les Byzantins. Peut-être faut-il attribuer ceci au climat humide de la France de langue d'oil et de l'Angleterre du sud (les grandes écoles de la fortification médiévale), climat offrant une certaine défense contre les effets du feu. Peut-être cela tiendrait-il au manque de certains produits essentiels: le naphte qui ne se trouve qu'au Moyen Orient, le soufre qu'un Italie, etc.

Les «réceptaires» des praticiens médiévaux s'occupent parfois des moyens de maîtriser ou de s'opposer aux feux de guerre. Ainsi la Mappae clavicula de efficiendo auro, recueil qui fait partie du manuscrit dit de Lucques ${ }^{26}$ copié vraisemblablement au temps de Charlemagne mais

${ }^{25}$ M. Mercler, Le feu grégeois, p. 92 et suiv.

${ }^{26}$ Le texte de la Mappae clavicula nous a été transmis par divers manuscrits: Biblioth. des Chanoines, Lucques, codex 490; Biblioth. Municipale de Sélestat, ms. n. 1153 bis (du X' $\mathrm{X}^{\circ}$ siècle); Biblioth. Nat. Paris, ms. latin n. ${ }^{\circ} 6830 \mathrm{~F}$ (du XII" dans le recueil intitulé «Opus agriculturae» de Palladius), etc. Sur l'histoire de ces manuscrits, cf. V. MorteT, Un formulaire du VIII siècle, in: «Bulletin Monumental», vol. 71 (1907), pp. 442-465. Le texte, d'après un manuscrit de Cheltenham 
dont la rédaction aurait eu lieu quelques deux ans auparavant, et dont le texte nous a été transmis aussi par des manuscrits du $\mathrm{X}^{\mathrm{u}}$ et du XII' siècles. La Mappae conseille de revêtir l'ouvrage à préserver de couches successives de cuir, de feutre, de laine, etc., humectées de vinaigre et d'urine fermentée, procédé aussi compliqué que difficile à mettre en oeuvre. Dans la plupart des cas on se contente de couvrir les charpentes avec du gazon, du fumier, du cuir ou — s'il s'agit d'une faible surface - avec des peaux de bêtes fraichement écorchées. Parfois ces charpentes sont crépies d'argile, de talc ou impregnées de solutions telles celles d'alun qui, en s'évaporant, déposent une sorte de péllicule protectrice ${ }^{27}$. Aulu Gelle, au $\mathrm{II}^{\mathrm{e}}$ siècle, note que lors du siège du Pirée par Sylla: «... une tour de bois, faite par les assiégés, fut enveloppée de toutes parts par les flammes et ne put brûler. Archélaus [chef des défenseurs] l'avait enduite d'alun» ${ }^{28}$. Au XIV ${ }^{\mathrm{e}}$ siècle, les Byzantins s'efforcent de brûler un vaisseau vénitien au moyen du feu grégeois mais «les Vénitiens accoutumés à leurs usages et qui avaient recouverts et entourés leur navire d'étoffes de laine imbibée de vinaigre, naviguèrent en toute sécurité» ${ }^{29}$.

Quant aux lanceurs ou manieurs de feu, aux «naffatin» des auteurs arabes, ils portent des vêtements ignifugés par une couche de talc, ce qui leur assure une protection suffisante.

Pour attaquer le feu lui-même, on se sert de vinaigre ou d'urine fermentée ou bien on essaye d'étouffer le feu sous de la cendre, de la terre, du sable; Ammien Marcellin, on l'a vu, indique ce dernier procédé à deux reprises. Notons en passant que dans l'Antiquité et au Moyen Âge, le mot vinaigre s'applique non seulement à l'acide acétique dilué mais à toutes sortes de liquides acides et même à des solutions comme la saumure qui laissent une couche incombustible. Quant à l'urine fermentée, sa teneur en ammoniaque en fait un dissolvant des graisses, que l'on pourait rapprocher de nos détergeants.

Il se pourait enfin que le désir de s'opposer aux effets des feux de guerre ait poussé les peuples méditerrannéens à substituer la pierre au bois dans les ouvrages militaires, hourdages en particulier, et l'enceinte de Montagnana, ville proche à Venise, aurait été modifiée dans ce sens

(du XII" siècle) a été publié par A. WAY (in: Arcbaeologia, London, 1847, vol. 32, pp. 183-254).

${ }_{27}$ Diverses formules arabes d'ignifugation sont données par $\mathrm{C}_{\mathrm{L}}$. CAHEN, Un traité d'armurrerie composé pour Saladin, in: «Bulletin d'études orientales», vol. 12 (Damas, 1947-1948), pp. 103-163.

${ }_{28}$ Aulu Gelle, Nuits attiques, liv. XV, chap. 1, éd. Nisard, Paris, Didot, 1875, p. 666 .

${ }^{29}$ M. Mercier, Du feu grégeois, p. 24. 
dès la fin du XIII" siècle $^{30}$. En fut-il de même dans quelque ouvrage français? La question mériterait d'être étudiée et peut-être nous sera-t-il donné quelque jour de nous y risquer.

\section{LES GAZ ASPHYXIANTS}

Les fumées et les gaz plus ou moins suffoquants ont été employés, depuis des temps reculés, pour arrêter un adversaire, surtout dans un lieu renfermé. Au siège d'Ambracia, en 190 av. J. C., les défenseurs chassent les Romains d'une galerie de mine en les enfumants par la combustion de plumes contenues dans une jarre et recouvertes de braises ${ }^{31}$. Mais on peut se servir aussi de ces moyens dans les combats à ciel ouvert. Au $\mathrm{X}^{\mathrm{c}}$ siècle, l'empereur byzantin Léon VI dit de lancer contre les vaisseaux ennemis «des pots pleins de chaux vive qui, en se brisant, répandent une épaisse fumée dont la vapeur suffoque et enveloppe d'obscurité l'ennemi» ${ }^{32}$. A la bataille de Tibériade ou de Hattin (1187), les Sarrazins mettent le feu à des broussailles et la fumée contribue à la déroute des Croisés luttant, toute la journée sous un climat torride. En 1216, au siège du château de Beaucaire, les défenseurs descendent jusqu'au pied des murs une sorte de sac contenant du soufre, de l'étoupe et des braises, les vapeurs sulfureuses qui se dégagent de la combustion, chassent les pionniers ennemis ${ }^{33}$. Sensiblement à la même époque, un manuscrit arabe, le Traité des stratagèmes, des guerres, de la prise des villes et de la défense des routes-frontières, indique l'emploi de feux de bois arrosé d'un naphte spécialement préparé et produisant des fumées asphyxiants ${ }^{3.4}$. Au XV siècle, l'allemand Konrad Kyeser parle des vapeurs nauséabondes qui se dégagent en jetant sur le feu du soufre, de la colophane, de la poix, des sabots de cheval, etc. ${ }^{35}$. En 1498, Menaut de

30 Renseignement aimablement communiqué par M. A. A. Weissmüller.

${ }^{31}$ S. ToY, A bistory of fortification from 3000 B. C. to 1700 A. D., London, Heinemann, 1955, pp. 36-37. Sa référence est: «Hero from Polybius, XXI, 28».

${ }^{32}$ Tactica, chap. $19, \$ 54$.

${ }^{33}$ La cbanson de la croisale albigeoise, laisse 164, vers 23-28, éd. B. MartinChabot, Paris, Champion-Les Belles Lettres, 1931-1961, vol. II, pp. 158-159.

${ }^{31}$ Les deux manuscrits de ce Traité, datés des environs de 1225 , conservés à la Biblioth. de l'Université Royale de Leyde (Cod. orient. n. ${ }^{\circ} 92$ et 499) diffèrent quelque peu entre-eux. Les recettes de gaz asphyxiants se trouvent reproduites in L. N. Bonaparte, Etudes sur le passé el l'avenir de l'artillerie, vol. III, pp. 8-14.

${ }_{35}$ KonRad Kyeser, Bellifortis. Il existe plusieurs manuscrits de cet ouvrage. Le texte du codex philosoph. 63 de la Biblioth. de l'Université de Göttingen a été édité facsimilairement, par Gotz Qunkg, London, Phaidon Press, 1968, 2 vols. Cf. aussi B. Gille, Les ingénieurs de la Renaissance, Paris, Hermann, 1964, p. 58. 
Guerre ou d'Aguerre, capitaine basque au service du roi de France, accuse le pape Alexandre Borgia d'avoir ou recours à des gaz asphyxiants pour tenter de réduire la garnison française du château d'Ostie ${ }^{36}$. Les «gaz», si largements employés pendant la guerre de 1914-1918, ne constituaient donc pas une nouveauté.

${ }^{36} \mathrm{G}$. Oux, Le pape Alexandre VI a-t-il employé les armes cbimiques?, in: Recueil de travaux offerts à $M$. Clovis Brunel, Paris, Société de l'École des Chartes, 1955, vol. I, pp. 321-334. 